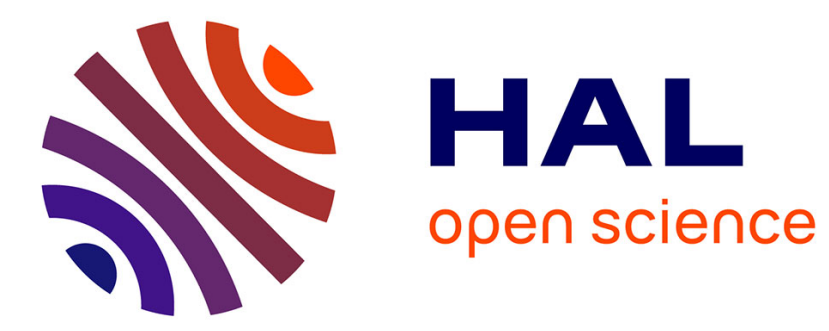

\title{
Mesures sur la précision des pointés longitudinaux
}

B. Dossier, P. Jacquinot, M. Maréchal, G. Pieuchard

\section{To cite this version:}

B. Dossier, P. Jacquinot, M. Maréchal, G. Pieuchard. Mesures sur la précision des pointés longitudinaux. J. Phys. Radium, 1951, 12 (4), pp.565-567. 10.1051/jphysrad:01951001204056501 . jpa-00234424

\section{HAL Id: jpa-00234424 https://hal.science/jpa-00234424}

Submitted on 1 Jan 1951

HAL is a multi-disciplinary open access archive for the deposit and dissemination of scientific research documents, whether they are published or not. The documents may come from teaching and research institutions in France or abroad, or from public or private research centers.
L'archive ouverte pluridisciplinaire HAL, est destinée au dépôt et à la diffusion de documents scientifiques de niveau recherche, publiés ou non, émanant des établissements d'enseignement et de recherche français ou étrangers, des laboratoires publics ou privés. 


\section{MESURES SUR LA PRÉGISION DES POINTÉS LONGITUDINAUX}

Par B. DOSSIER, P. JACQUINOT, M. MARÉCHAL et G. PIEUCHARD

Dans une précédente lettre [1], nous avons proposé des répartitions d'amplitudes pupillaires permettant de réduire la profondeur de foyer d'un objectif. Nous donnons ici les résultats de mesures visuelles et d'enregistrements photométriques faits avec les diaphragmes proposés $\left(\mathrm{C}_{0}\right.$ et $\left.\mathrm{L}_{0}\right)$, comparés aux diaphragmes classiques uniformes $\mathrm{C}$ (circulaire) et $\mathrm{L}$ (rectangulaire), ces derniers étant, en outre, comparés entre eux. La confrontation des résultats visuels et des enregistrements montre que l'œil peut être sensible, pour juger de la meilleure mise au point, à la variation d'autres grandeurs que l'éclairement central, qui a servi de base au calcul exposé précédemment.

I $^{\circ}$ Pointés visuels. - Deux séries d'expériences (Institut d'Optique et Bellevue) ont été faites avec deux systèmes optiques différents ayant tous deux une très faible ouverture $\left(\Omega=\mathrm{I} / \mathrm{IOO}^{\mathrm{e}}\right)$, de façon à rester dans le domaine de la diffraction pure, et une 
distance focale-suffisante (respectivement 2 et $10 \mathrm{~m}$ ), de façon à mesurer facilement les écarts de mise au point; on pouvait viser soit un point, soit une ligne lumineuse, en lumière monochromatique. Les défauts de mise au point peuvent s'exprimer soit en différence de chemin optique entre rayons centraux et marginaux, soit en unités Rayleigh $\left(R=\frac{2 \lambda}{\Omega^{2}}\right)$ correspondant à une différence de marche de $\frac{\lambda}{4}$. Pour chaque type de diaphragme les écarts quadratiques moyens $\bar{z}$ de mise au point ont été calculés d'après quelques centaines de mesures réparties entre plusieurs observateurs. Dans le cas du diaphragme $L_{0}$ on visait non seulement la position du foyer, mais aussi le plan défocalisé de Rayleigh dans lequel on obtient un système de franges d'interférence deux fois plus serrées et dont les alternances d'intensité s'inversent, comme l'a montré Zernike [2], quand on passe par cette position (position sensible). Le tableau donne les écarts $\bar{z}$, ainsi que les gains de précision $G$ obtenus par rapport au diaphragme circulaire uniforme. Dans le cas de la comparaison entre diaphragmes circulaires et diaphragmes linéaires on donne deux valeurs de $G$, la première $G_{1}$ supposant un diaphragme linéaire de largeur totale égale au diamètre du diaphragme circulaire, la deuxième $G_{2}$ supposant un diaphragme linéaire inscrit dans un carré de diagonale égale au diamètre du diaphragme circulaire (condition de luminosité maximum). La supériorité du diaphragme $\mathrm{L}_{0}$ utilisé à la position sensible est évidente; elle est dûe à ce que le phénomène observé n'est pas stationnaire, contrairement à tous les autres cas. Il semble cependant que le diaphragme $\mathrm{C}_{0}$ soit plus agréable à utiliser, tout en conservant un gain de précision $G_{2}=1,7$ dans la visée d'une ligne comme dans celle d'un point (ce que le calcul ne faisait pas prévoir).

\begin{tabular}{|c|c|c|c|c|c|}
\hline Souree & & Écrans. & Écarts $\bar{z}$. & $i_{1}$. & $a_{2}$. \\
\hline P'oint & $t \ldots \ldots \ldots$ & $C$ & $\frac{\lambda}{3 o}$ & I & - \\
\hline 》) & $\ldots \ldots \ldots$ & $C_{0}$ & $\frac{\lambda}{50}$ & $\mathbf{I}, 7$ & I, 7 \\
\hline Ligne & $e \ldots \ldots \ldots \ldots$ & $C$ & $\frac{\lambda}{30}$ & I & - \\
\hline " & $\ldots \ldots \ldots \ldots$ & $C_{0}$ & $\frac{\lambda}{50}$ & $\mathrm{I}, 7$ & I, 7 \\
\hline ") & $\ldots \ldots \ldots$ & $L$ & $\frac{\lambda}{50}$ & $\mathrm{I}, 7$ & o, 8 \\
\hline " & . & $\left\{\begin{array}{c}L_{0} \\
(\text { foyer })\end{array}\right\}$ & $\frac{\lambda}{70}$ & 2,3 & I, I \\
\hline ") & & $\left\{\begin{array}{c}L_{0} \\
(\text { position } \\
\text { sensible })\end{array}\right\}$ & $\frac{\lambda}{200}$ & 6,7 & 3,8 \\
\hline
\end{tabular}

$2^{\circ}$ Enregistrements. - La répartition d'éclairements dans l'image d'une fente lumineuse a été enregistrée en explorant cette image avec une autre fente très fine. Le système enregistreur photoélectrique était celui du grand spectrographe de Bellevue, qui comporte un système à double faisceau équilibré par servomécanisme; l'optique, la même que pour les pointés visuels, était également celle du spectro- graphe, dont le prisme était supprimé. Les figures I et 2 représentent par exemple les courbes obtenues pour différentes valeurs de la défocalisation $z$ pour les diaphragmes $\mathrm{L}$ et $\mathrm{C}_{0}$. De telles figures ont été

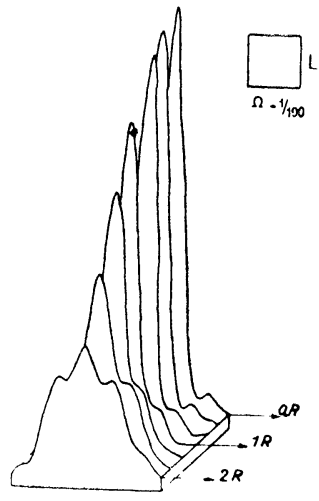

Fig. I.

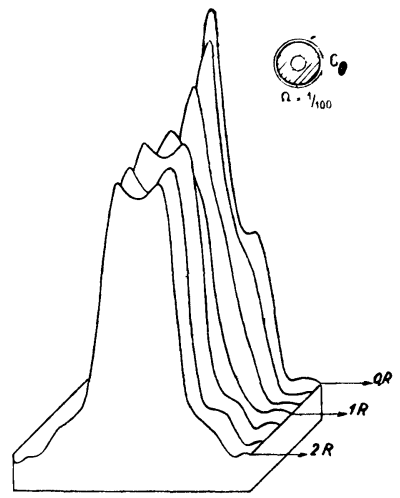

Fig. 2 .

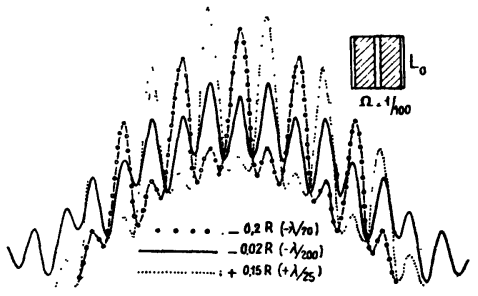

Fig. 3.

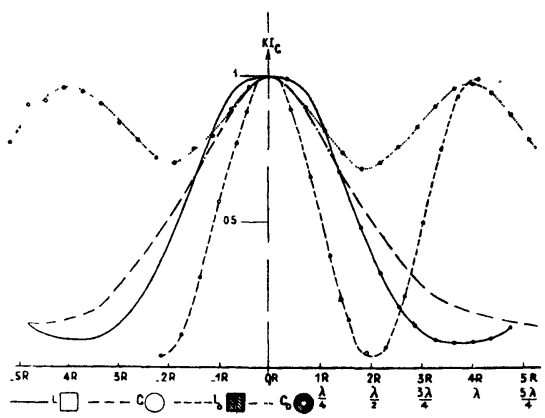

Fig. 4 . 
enregistrées pour tous les types de diaphragmes et la figure \& représente les variations (normalisées) de l'éclairement central. L'efficacité de $L_{0}$ au foyer s'explique suffisamment par cette variation, mais non celle de $\mathrm{C}_{0}$ avec fente. Pour l'expliquer on peut déterminer et comparer entre elles, d'après la figure 5,

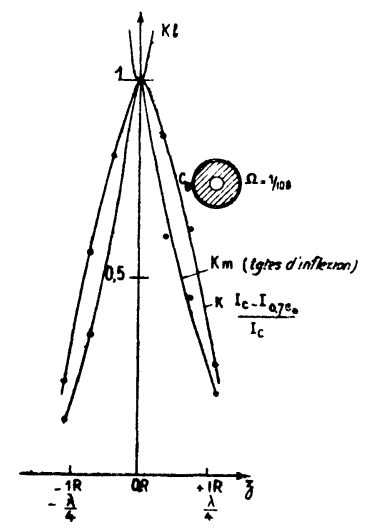

Fig. 5 .

les variations (normalisées) des différentes grandeurs auxquelles l'œil pourrait être sensible, largeur du pic central, pente d'inflexion de ce pic, contraste $\frac{I_{c}-I_{x}}{I_{c}}$ entre le centre et l'“ épaulement " voisin; c'est cette dernière grandeur qui semble intervenir. La figure 3 représente, sous une forme différente, trois courbes d'éclairement avec $\mathrm{L}_{0}$ et pour trois positions très peu différentes $(-0,2 R$; $+0,02 R$ et $+0,15 R) d$ 'une position sensible; la figure 6 , qui montre les variations

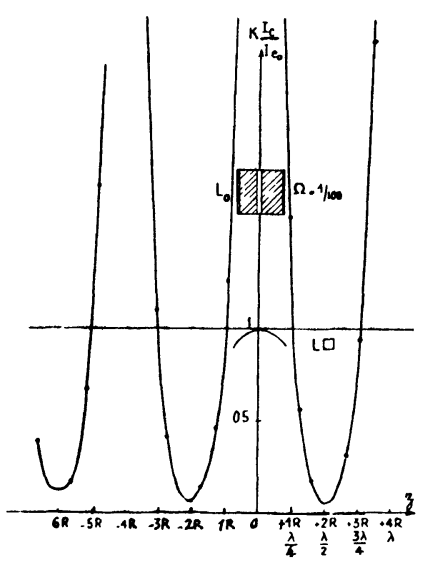

Fig. 6 .

du contraste entre deux franges voisines, permet de comparer cette variation non stationnaire à celle, stationnaire, de l'éclairement central dans le cas du diaphragme $\mathrm{L}$ et permet de rendre compte de la sensibilité du dispositif. D'après les enregistrements de la figure 3 on peut déterminer une position sensible avec une erreur inférieure à $\frac{R}{250}$ (correspondant à une différence de marche de $\left.\frac{\lambda}{\mathrm{I} 000}\right)$, compte tenu des fluctuations ou infidélités de l'enregistrement.
Signalons enfin que nous avons expérimenté par ce procédé l'éclairage cohérent dissymétrique d'une fente de largeur convenable (voir [3] et [4]), tel que la pupille (ici rectangulaire) soit couverte par la demifrange centrale et la première frange latérale de la

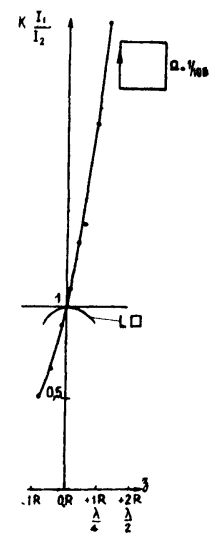

Fig. 7 .

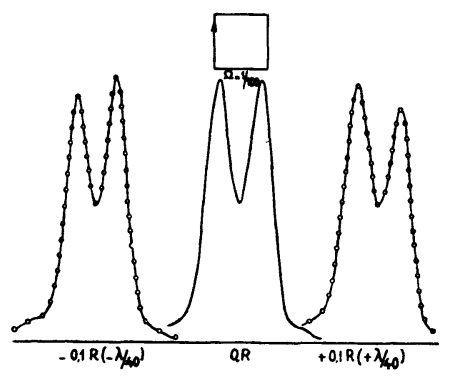

Fig. 8 .

figure de diffraction de la fente source. La figure 8 donne les enregistrements faits au foyer et de part et d'autre $( \pm 0, \mathrm{I} R)$ et la figure 7 le contraste entre les deux franges, comparé à la variation d'éclairement central du cas classique. Ce contraste est un nouvel exemple de grandeur non stationnaire : toutefois il varie six fois moins vite que le contraste obtenu avec $L_{0}$ à la position sensible.

[1] B. Dossier, P. JAcQuinot, A. Maréchal et G. Pieughard - J. Physique Rad. i 95 I, 12, I 42.

[2] F. Zernike - J. Opi. Soc. Amer., i 95o, 40, no 5, 326-328.

[3] M. Duffieux et Coll. - Ann. Physique, 1944, 19, 380.

[4] B. Dossier et P. JAcQuinot - Journal des Recherches $d u$ C.N.R.S., I 950,12 , г $23-138$.

Manuscrit reçu le i 6 février $195 \mathrm{I}$. 